\title{
Three-Dimensional Visualization and Characterization of Polymeric Self-Assemblies by Transmission Electron Microtomography
}

Published as part of the Accounts of Chemical Research special issue "Direct Visualization of Chemical and SelfAssembly Processes with Transmission Electron Microscopy".

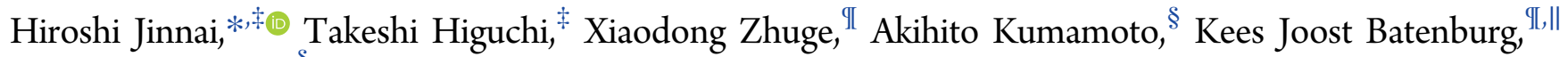
and Yuichi Ikuhara ${ }^{\S}$

${ }^{\ddagger}$ Institute of Multidisciplinary Research for Advanced Materials, Tohoku University, 2-1-1 Katahira, Aoba-ku, Sendai 980-8577, Japan

${ }^{\text {II }}$ Centrum Wiskunde and Informatica, Amsterdam 1098 XG, The Netherlands

${ }^{\S}$ Institute of Engineering Innovation, School of Engineering, The University of Tokyo, 2-11-16 Yayoi, Bunkyo-ku, Tokyo 113-8656, Japan

"Mathematical Institute, Leiden University, RA Leiden 2300, The Netherlands

Supporting Information

CONSPECTUS: Self-assembling structures and their dynamical processes in polymeric systems have been investigated using three-dimensional transmission electron microscopy (3D-TEM). Block copolymers (BCPs) self-assemble into nanoscale periodic structures called microphase-separated structures, a deep understanding of which is important for creating nanomaterials with superior physical properties, such as high-performance membranes with well-defined pore size and high-density data storage media. Because microphase-separated structures have become increasingly complicated with advances in precision polymerization, characterizing these complex morphologies is becoming increasingly difficult. Thus, microscopes capable of obtaining 3D images are required. In this article, we demonstrate that 3D-TEM is an essential tool for studying BCP nanostructures, especially those self-assembled during dynamical processes and under confined conditions.

The first example is a dynamical process called order-order transitions (OOTs). Upon changing temperature or pressure or applying an external field, such as a shear flow or electric field, BCP nanostructures transform from one type of structure to another. The OOTs are examined by freezing the specimens in the middle of the OOT and then observing the boundary structures between the preexisting and newly formed nanostructures in three-dimensions. In an OOT between the bicontinuous double gyroid and hexagonally packed cylindrical structures, two different types of epitaxial phase transition paths are found. Interestingly, the paths depend on the direction of the OOT. The second example is BCP self-assemblies under confinement that have been examined by 3D-TEM. A variety of intriguing and very complicated 3D morphologies can be formed even from the BCPs that self-assemble into simple nanostructures, such as lamellar and cylindrical structures in the bulk (in free space). Although 3D-TEM is becoming more frequently used for detailed morphological investigations, it is generally used to study static nanostructures. Although OOTs are dynamical processes, the actual experiment is done in the static state, through a detailed morphological study of a snapshot taken during the OOT. Developing time-dependent nanoscale 3D imaging has become a hot topic. Here, the two main problems preventing the development of in situ electron tomography for polymer materials are addressed. First, the staining protocol often used to enhance contrast for electrons is replaced by a new contrast enhancement based on chemical differences between polymers. In this case, no staining is necessary. Second, a new 3D reconstruction algorithm allows us to obtain a high-contrast, quantitative $3 \mathrm{D}$ image from fewer projections than is required for the conventional algorithm to achieve similar contrast, reducing the number of projections and thus the electron beam dose. Combining these two new developments is expected to open new doors to $3 \mathrm{D}$ in situ real-time structural observation of polymer materials.

\section{INTRODUCTION}

Polymer materials are all around us in our daily lives. Many polymers are multicomponent systems composed of multiple polymer species. The classic multicomponent system is a mixture of two or more constituent polymers: a polymer blend. Because of the repulsive interactions between the constituents, multicomponent polymer materials often undergo phase separation. There has been a significant increase in the number of studies characterizing this kind of phase-separated structure

Received: February 28, 2017

Published: May 19, 2017 
over the past couple of decades, driven by academic interest in complex fluids (of which polymeric systems are an example) and the endless need for new materials in industrial applications.

Self-assembling structures and their dynamical processes in polymeric systems have been investigated by using threedimensional transmission electron microscopy (3D-TEM). Block copolymers (BCPs) self-assemble into nanoscale periodic structures called microphase-separated structures, a deep understanding of which is important to create nanomaterials with superior physical properties, including high-performance membranes with well-defined pore size and high-density data storage media. Because microphase-separated structures have become increasingly complicated as control of polymerization has become increasingly precise, identifying these complex morphologies is becoming increasingly difficult. Thus, microscopes capable of obtaining 3D images are required. In this article, we demonstrate that $3 \mathrm{D}$-TEM is an essential tool for studying BCP nanostructures, especially those self-assembled during dynamical processes and under confined conditions.

The first example is a dynamical process called order-order transition (OOT). Upon changing temperature or pressure or applying an external field, such as a shear flow or electric field, BCP nanostructures transform from one type of structure to another. The OOTs are examined by freezing the specimens during the OOT and then observing the boundary structures between the preexisting and newly formed nanostructures in three-dimensions (3D). In an OOT between bicontinuous double gyroid (DG) and hexagonally packed cylindrical structures, there are two epitaxial phase transition paths that depend on the direction of the OOT. The second example is BCP self-assemblies under confinement, which were also examined by $3 \mathrm{D}$-TEM. It was found that a variety of intriguing and complicated 3D morphologies are formed, even from the BCPs that self-assemble into simple nanostructures, such as lamellar and cylindrical structures, in the bulk (in free space).

Although 3D-TEM is becoming more frequently used for detailed morphological investigations, it is generally used to study nanostructures in a static state. Although OOT is a dynamical process, the morphological study of a snapshot during the OOT is performed in the static state. Developing time-dependent nanoscale 3D imaging has become the focus of significant research. Here, two major problems preventing the development of in situ electron tomography for polymer materials are addressed. First, the staining protocol often used to enhance contrast for electrons is replaced by a new contrast enhancement based on chemical differences between polymers. In this case, no staining is necessary. Second, a new 3D reconstruction algorithm allows us to obtain a high-contrast, quantitative $3 \mathrm{D}$ image from fewer projections than those required for the conventional algorithm to achieve similar contrast, reducing the number of projections and thus the electron beam dose. Combining these two developments is expected to open new doors to $3 \mathrm{D}$ in situ real-time structural observation of polymer materials.

In industry, phase-separated polymer systems are used to create nanomaterials with superior physical properties and thus need to be well understood. Owing to the high regularity of microphase-separated structures of BCPs, they are increasingly important in creating high-tech products. These structures are often used as templates for high-performance membranes with well-defined pore size ${ }^{1}$ and for lithography masks to fabricate high-density data storage media. ${ }^{2}$ Similarly, the optical and electrical properties of nanomaterials can be controlled by distributing nanoparticles precisely by using microphaseseparated structures as nanotemplates. ${ }^{3,4}$ Although grain boundary structures are often considered as defects, these structures sometimes improve material properties, ${ }^{5}$ and their morphological features and formation processes should be investigated.

Previous morphological studies of multicomponent polymeric materials have employed various microscopic and scattering methods. Transmission electron microscopes (TEM), scanning electron microscopes, and atomic force microscopes are commercially available and widely used for nanoscale structure observation. The biggest advantage of microscopy is that various morphologies can be observed intuitively in real space. Phase-separated structures, and particularly microphase-separated structures, have become increasingly complicated as control of polymerization has become more precise, and identification of these complex morphologies is becoming increasingly difficult. Micrographs taken by conventional microscopes, such as TEM, are often inconclusive because conventional microscopes take twodimensional (2D) (transmitted or surface) images of threedimensional (3D) objects. The development of microscopes that can acquire $3 \mathrm{D}$ images is the logical next step.

Laser-scanning confocal microscopy (LSCM) was developed in the 1980s and first became widely used in the biological community for direct 3D observation of cell morphologies. Over time, it eventually became popular in the materials community. LSCM was first used to observe the bicontinuous morphology of a polymer mixture during spinodal decomposition. ${ }^{6-8} \mathrm{X}$-ray computerized tomography (CT) is another technique for $3 \mathrm{D}$ imaging that has a spatial resolution similar to that of LSCM, but no better than a few micrometers. The biggest advantage of X-ray CT over LSCM is the strong penetrative power of the X-rays that allows it to be readily used for opaque materials. It has recently been used to observe dynamical processes in fiber-reinforced plastic under uniaxial extension in which the orientation of fibers and formation of voids were clearly imaged and correlated with mechanical properties. ${ }^{9}$ For extremely fine resolution, the atom probe fieldion microscope was developed to allow atom probe tomography with truly atomic scale precision, ${ }^{10}$ though few studies have applied this type of microscopy to polymers.

Another technique is needed to cover intermediate resolutions $(\sim 1 \mathrm{~nm})$ with a field of view of hundreds of nanometers for characterizing polymeric structures. 3D-TEM is an emerging technique for 3D structural observations based on TEM in polymer nanotechnology. In a 3D-TEM experiment, the specimen is tilted with respect to the electron beam in the TEM column. A 3D image is obtained based on CT of the transmitted images at different angles, typically over the range of $\pm 70^{\circ} .^{11-13} 3 \mathrm{D}$-TEM is the only method that provides the geometrical features of nanoscale 3D morphologies. For example, the genus, a measure characterizing the complexity of a $3 \mathrm{D}$ structure, can be measured from 3D images based on differential geometry. ${ }^{14}$ The first study using 3D-TEM was published in 1988 by Spontak et al. ${ }^{15}$ Although 3D-TEM was not used extensively at first, it is increasingly being used to investigate $\mathrm{BCP}$ morphologies.

In this Account, we focus on direct visualization of polymeric self-assembled nanostructures and their processes in BCPs by 3D-TEM. This article is organized in the following manner: In section 2, we describe $3 \mathrm{D}$ observations of dynamical process of 
$\mathrm{BCP}$ for an OOT in which a BCP nanostructure transforms to another type of nanostructure upon changing temperature, pressure, or another parameter. In section 3, BCP selfassemblies under confinement are described. It is found that complicated and intriguing nanostructures are formed when the space for BCP self-assembly is limited. 3D-TEM is found to be an essential tool for studying BCP nanostructures under confinement. In section 4, we describe 3D-TEM for unstained BCP in which we propose a novel method for generating contrast with electrons using scanning transmission electron microscopy (STEM) with a newly developed reconstruction technique. The contrast is because of chemical differences between the two constituent polymers. This kind of unstained imaging is essential for in situ time-resolved nanoscale 3D imaging for dynamical processes in polymeric systems.

\section{3D STRUCTURAL ANALYSIS OF OOTS}

The main advantage of $3 \mathrm{D}$-TEM is that it does not require periodic structures (such as protein crystallography) or atomically identical copies; hence, it can be used to characterize unique 3D structures. It has been demonstrated that 3D-TEM is one of the most powerful methods for investigating static polymer nanostructures, especially complex ones. Unlike other microscopy techniques such as light microscopy, 3D-TEM samples are microtomed, which limits this technique to observations of fixed 3D morphologies. In some cases, however, dynamical processes of polymer nanostructures can be studied from 3D snapshots.

BCPs undergo OOTs, which often exhibit an epitaxial relationship between the morphology of two phases. ${ }^{16-25} \mathrm{We}$ show two examples of OOTs from and to the DG structure, which is interesting because of the structural complexity of the DG phase. ${ }^{13}$

Figure 1 shows a 3D image and digitally sliced images of the DG/hexagonally packed cylinder (HEX) transitional structure of a poly(styrene-block-isoprene) (SI) BCP. ${ }^{26}$ The hexahedron of dimensions $400 \times 200 \times 80 \mathrm{~nm}(X, Y, Z)$ in the figure represents the rectangular box in the TEM image in Figure $1 b$, where $Z$ is the direction of incident electron beam and $X-Y$ is the film plane (i.e., substrate surface). Figure $1 \mathrm{~b}$ clearly shows the grain boundary region of the coexisting DG and HEX phases. The DG phase exhibits a wagon-wheel-shaped structure that is characteristic of the $\{111\}_{\mathrm{DG}}$ plane, whereas HEX cylinders are oriented along the $X$-axis. Several digitally sliced images are obtained of cross sections of the hexahedron to understand more deeply the 3D nanostructure of the coexisting morphologies of DG and HEX. Figure le shows an image of the HEX grain perpendicular to the cylinder axis, and Figure if shows an image of $\{220\}_{\mathrm{DG}}$, which is orthogonal to both $\{121\}_{\text {DG }}$ and $\{111\}_{\text {DG }}$.

In this example, the HEX cylinder axis is perpendicular to $\{220\}_{\mathrm{DG}}$ and parallel to both $\{121\}_{\mathrm{DG}}$ and $\{111\}_{\mathrm{DG}}$. Figure 1c shows the coexisting $\{100\}_{\mathrm{HEX}}$ and $\{121\}_{\mathrm{DG}}$, and Figure $1 \mathrm{~d}$ shows a magnified version of the image in Figure $1 \mathrm{~b}$. The former digital slice clearly shows coexisting $\{110\}_{\mathrm{HEX}}$ and $\{111\}_{\text {DG }}$.

Figure 2 shows schematic images of how the HEX cylinders evolve from the DG structure. According to Matsen's prediction, ${ }^{19}\{121\}_{\mathrm{DG}},\{220\}_{\mathrm{DG}}$, and $\{111\}_{\mathrm{DG}}$ are converted to $\{100\}_{\mathrm{HEX}},\{110\}_{\mathrm{HEX}}$, and $\{001\}_{\mathrm{HEX}}$, respectively. The interphase planes are $\{220\}_{\mathrm{DG}}$ and $\{110\}_{\mathrm{HEX}}$, and the cylinders meet the $\{220\}_{\text {DG }}$ plane parallel (side-on) at the grain boundary between DG and HEX. The projection along the
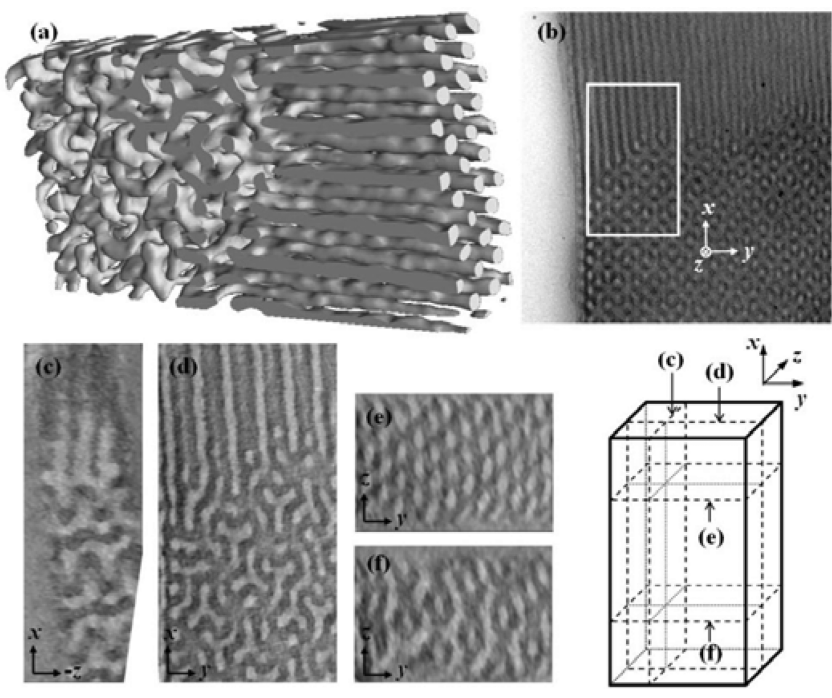

Figure 1. (a) 3D reconstructed image of the coexisting structure of DG and HEX. The dimensions of the hexahedron are $400 \times 200 \times 80$ $\mathrm{nm}(X, Y, Z)$. Only the polystyrene (PS) domain is shown. (b) TEM image of the sample region from which the $3 \mathrm{D}$ image was reconstructed (boxed area). (c-f) Cross-sectional images are shown in the rectangular solid in the lower right, where $Z$ is the direction of the incident electron beam and $X-Y$ is the film plane. The polyisoprene (PI) block was stained with $\mathrm{OsO}_{4}$ and appears darker. $3 \mathrm{D}$ observations by TEM and 3D-TEM were carried out using a JEM2200FS system (JEOL Co., Ltd., Japan) operating at $200 \mathrm{kV}$, details of which can be found elsewhere. ${ }^{26}$ Adapted with permission from ref 13 . Copyright 2010 American Chemical Society.

$\langle 111\rangle_{\text {DG }}$ direction has 3-fold symmetry (Figure 2a), and the loci corresponding to the HEX cylinder axes are indicated by small circles. These are not structurally uniform and have two different types. At the loci indicated by dotted circles, tripodal skeletons of two single gyroids are connected to form a HEX cylinder. This conversion has been proposed by Matsen. ${ }^{19}$ In the transitional structure observed in Figure 1, the HEX cylinders form along the $\langle 220\rangle_{\mathrm{DG}}$ direction. The symmetry of the $\{220\}_{\mathrm{DG}}$ projection is slightly distorted from the right hexagon (Figure 2b). The loci of cylinders are indicated by circles. In contrast to the above $\langle 111\rangle_{\mathrm{DG}} \rightarrow$ cylinder axis mechanism, ${ }^{19,23,27}$ all cylinders were formed through a topologically equivalent DG skeletal path. In this case, $\{121\}_{\mathrm{DG}},\{111\}_{\mathrm{DG}}$, and $\{220\}_{\mathrm{DG}}$ are converted to $\{100\}_{\mathrm{HEX}}$ $\{110\}_{\mathrm{HEX}}$ and $\{001\}_{\mathrm{HEX}}$, respectively. The interphase planes are $\{220\}_{\mathrm{DG}}$ and $\{001\}_{\mathrm{HEX}}$, and the cylinders meet the $\{220\}_{\mathrm{DG}}$ plane perpendicularly (head-on) at the boundary. Not only did this unexpected type of epitaxial transition occur, but the expected transitions, such as those predicted by Matsen, ${ }^{19}$ were not observed at all. Interestingly, however, when the direction of phase transition was inverted, from HEX to DG, a side-on epitaxial relationship was also observed as well as the head-on relationship. $^{28}$

A distinct feature of this example of OOT is the thin film structure in which a BCP thin layer (with thickness ranging from a few hundred nanometers to a few micrometers) is on a $\mathrm{Si}$ substrate. Surface interactions of BCPs may play a more important role in thin film OOTs than in bulk OOTs. Park et al. reported that hexagonally perforated layers ${ }^{29}$ are converted to $\{121\}_{\mathrm{DG}}$ in thin films $\mathrm{s}^{22,30}$ in the OOT from hexagonally perforated lamellar (HPL) to DG structures, whereas this kind of orderly transition is not observed in shear-oriented bulk. ${ }^{17,18}$ 


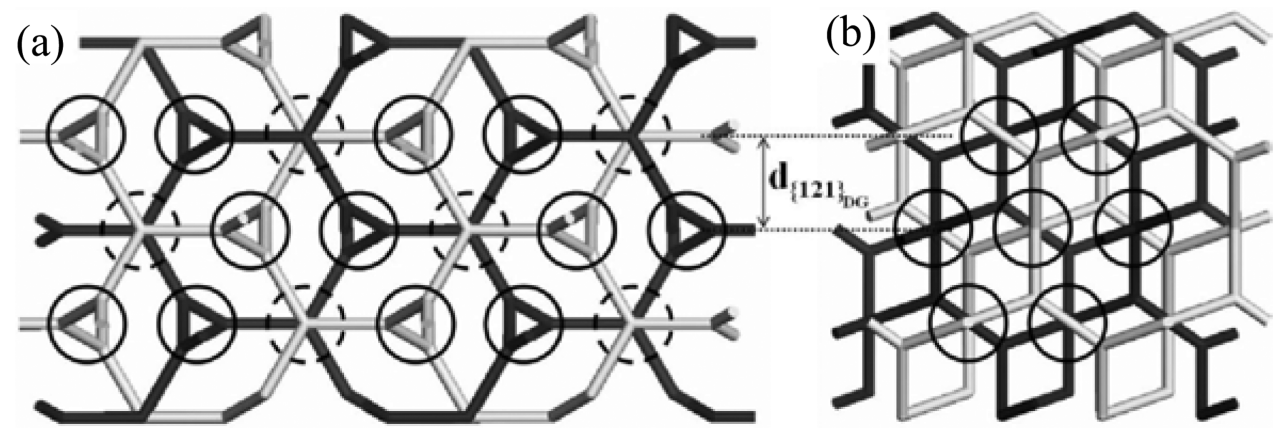

Figure 2. (a) Simulated projection along $\langle 111\rangle_{\mathrm{DG}}$. The two single gyroids are represented by different shades of gray. The loci of the cylinder axes are indicated by circles with solid and dotted circles representing different types of loci. (b) Simulated projection along $\langle 220\rangle_{\mathrm{DG}}$. The loci of the cylinder axes are indicated by circles. In this $\langle 220\rangle_{\mathrm{DG}} \rightarrow$ cylinder axis mechanism, all loci are equivalent. Adapted with permission from ref 13 . Copyright 2010 American Chemical Society.

Figure 3 shows a 3D image of the boundary between the HPL and DG morphologies. Every other HPL layer was beautifully

(a)

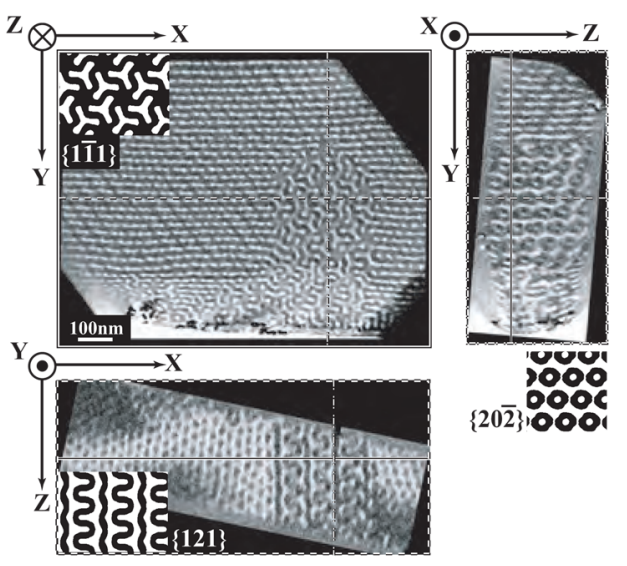

(b)

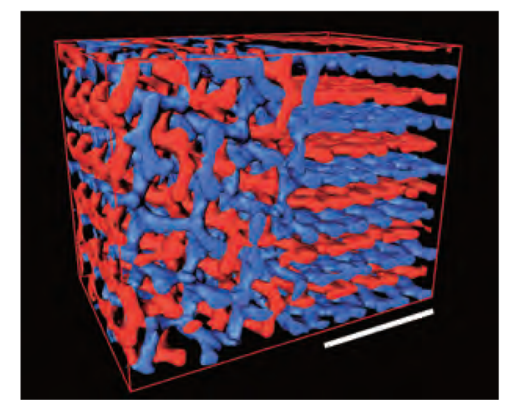

Figure 3. (a) Orthogonal views of the 3D reconstruction of the OOT. As shown in each cross section, the PS microdomain (white domain) of the DG exhibits a characteristic crystallographic plane, demonstrating that the OOT is epitaxial. The insets in the cross sections show corresponding patterns of DG viewed from each crystallographic plane indicated by Miller indices. (b) 3D morphology of SI BCP during OOT from HPL (right) to DG (left). The number-average molecular weight and polydispersity index of SI are $M_{\mathrm{n}}=37,000$ and $M_{\mathrm{w}} / M_{\mathrm{n}}=$ $1.01\left(M_{\mathrm{w}}\right.$ is the weight-average molecular weight $)$, respectively. The volume fraction of PI is 0.62 . Blue and red microdomains both consist of PS and are continuous through the boundary. Scale bar is $100 \mathrm{~nm}$.

connected to one of the two nonintersecting DG networks. As demonstrated in Figure $3 \mathrm{~b}$, an epitaxial relation can be found: $\{111\}_{\mathrm{DG}},\{121\}_{\mathrm{DG}}$, and $\{220\}_{\mathrm{DG}}$ planes are at the boundary where the $\{121\}_{\text {DG }}$ plane changes to HPL layers. ${ }^{30}$

Mareau et al. also reported the OOT between DG and HPL in the bulk. In their study, HPL layers were connected to
$\{111\}_{\mathrm{DG}}{ }^{31}$ which is incompatible with the epitaxial relationship between the HPL layers and $\{121\}_{\text {DG }}$ found in thin films. ${ }^{22}$ Accordingly, the OOTs in BCPs are not well understood and still need to be investigated in more detail. ${ }^{26,27,30}$ Although 3DTEM provides information about the epitaxial relations between the pre-existing and newly formed microdomains, it would be ideal to observe structural evolution during OOT in 3D. To the best of our knowledge, such multidimensional electron microscopy, that is, time-resolved 3D nanoscale imaging, has not been successful, at least in soft materials. A critical reason is the staining protocol often used to enhance contrast, as discussed later in section 4.

\section{MORPHOLOGIES OF BCP UNDER CONFINEMENT}

Microphase-separated structures in confined geometries have also received much attention because BCPs form morphologies that differ from those of bulk films due to spatial constraints. Various types of confinement have been reported, which have different dimensionalities and surface properties. ${ }^{32,33}$ In any confinement, surfaces separating the space where copolymers phase separate from the outside environment also play an important role. When the confinement matrix interacts equally with the constituent polymers of BCPs, both block chains appear at the interface, which is called a neutral surface. Conversely, matrices are fully covered by one constitute polymer when the matrices strongly interact with the segment. For the desired interfaces of matrices to be achieved, the surfaces of materials used for confinement spaces have been modified with self-assembled monolayers ${ }^{34}$ and polymer brushes. ${ }^{35}$ Random copolymers are grafted onto the surfaces of hard confinement matrices to prepare neutral surfaces. ${ }^{36}$

Dimensionality is another crucial factor for categorizing the confinement systems for microphase-separated structures. Onedimensional (1D) confinement is a thin film in which the substrate surface can be chemically modified to induce wetting of one of the phase-separated domains inside the film, ${ }^{37,38}$ which is known as chemical asymmetry. The film thickness can also act as the confinement. In BCP thin films, if the film thickness is not a multiple integer of the period microphaseseparated domain, physical asymmetry occurs, and the film surface is not completely flat because frustration occurs to accommodate these periodic domains. For example, not only the orientation of cylindrical structures but also morphologies change with different film thicknesses. ${ }^{39}$ Note that perforated lamellar structures have been induced by the confinement effect. $^{38}$ 

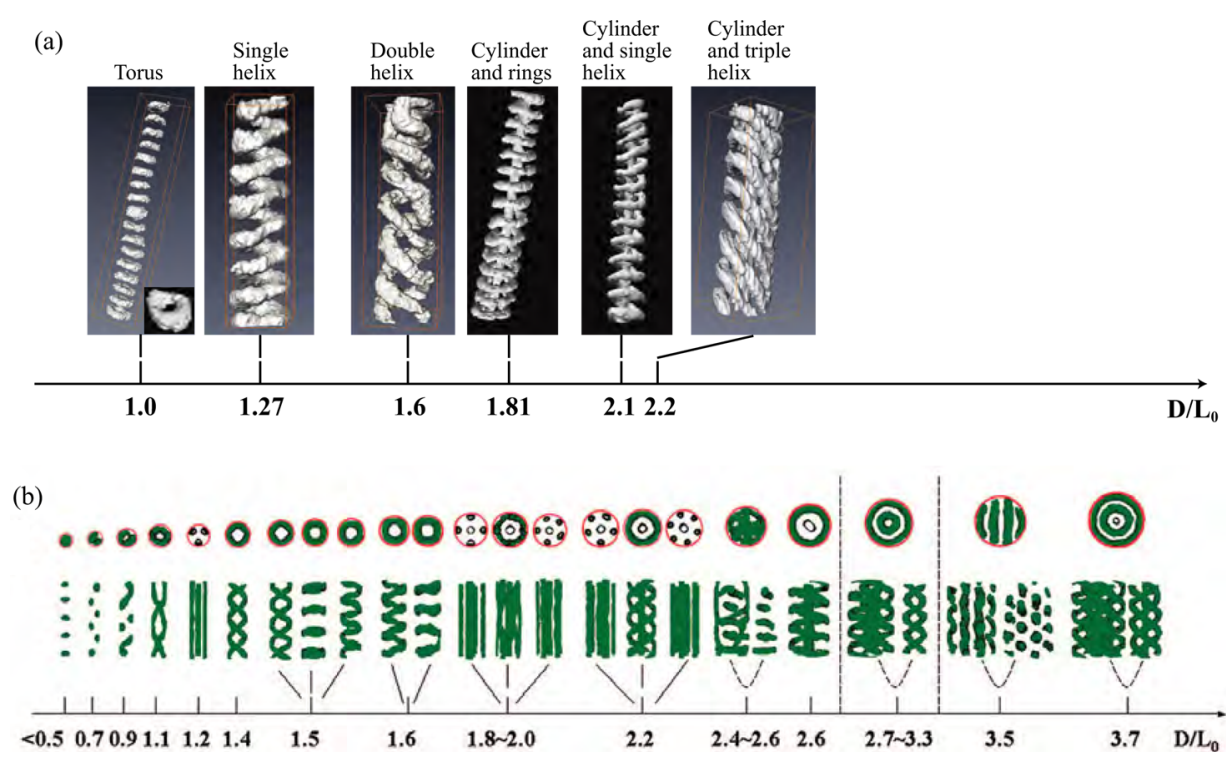

Figure 4. (a) 3D structures of cylinder-forming PS- $b$-PBD in AAO cylindrical pores of different diameters. Only the PBD phase is shown. The morphologies are organized as a function of $D / L_{0}$. Adapted with permission from ref 44. Copyright 2009 American Chemical Society. (b) Simulated structures of cylinder-forming $\mathrm{AB}$ diblock copolymers under cylindrical $2 \mathrm{D}$ confinement for different $D / L_{0}$ when the majority A constituent block preferentially wets the surface of the $2 \mathrm{D}$ confinement. Only minority B phases are shown. Adapted with permission from ref 45 . Copyright 2009 American Chemical Society.

(a)

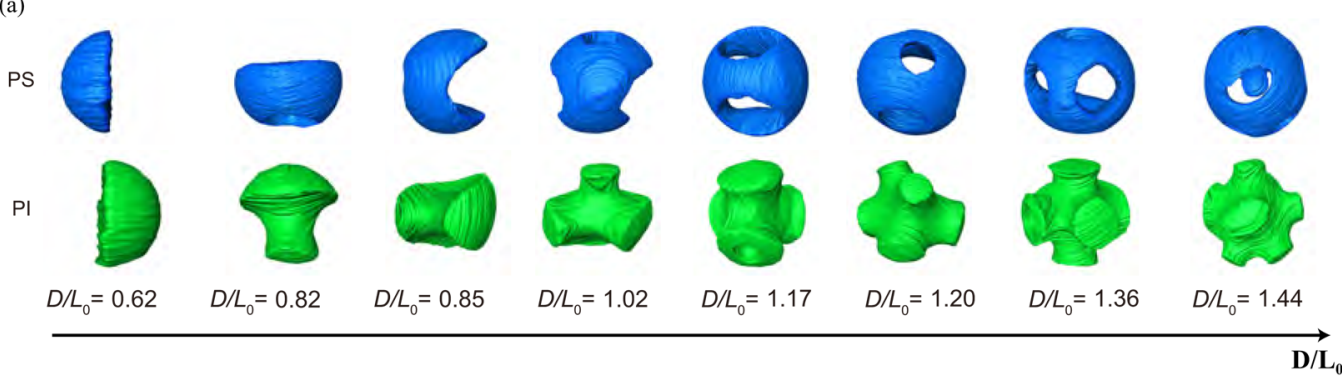

(b)

PS

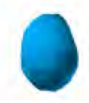

PI

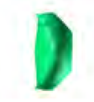

$D / L_{0}=1.5$
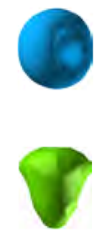

$D / L_{0}=1.7$
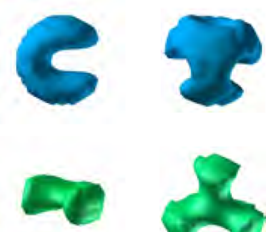

$D / L_{0}=1.7$

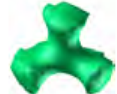

$D / L_{0}=2.1$
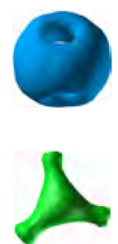

$D / L_{0}=2.1$
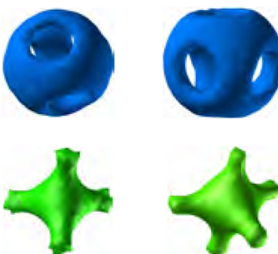

$D / L_{0}=2.4$

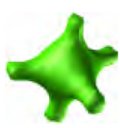

$D / L_{0}=2.5$
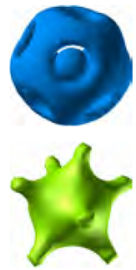

$D / L_{0}=3.0$

${\mathrm{D} / \mathrm{L}_{0}}$

Figure 5. (a) 3D structures of SI nanoparticles with different $D / L_{0}$ obtained by $3 \mathrm{D}-\mathrm{TEM}$. (b) Simulated structures of a diblock copolymer in spherical confinement. Adapted with permission from ref 53. Copyright 2016 Wiley-VCH Verlag GmbH \& Co.

Examples of 2D confinement include the inside of electrospun fibers, ${ }^{40,41}$ internal cylindrical pores in anodic aluminum oxide (AAO), ${ }^{42-44}$ which have two boundary conditions $d_{x}$ and $d_{y}$ corresponding to the diameters of the cylinders in the $x$ and $y$ directions. Because the structures in 2D confinement systems are much more complicated than in $1 \mathrm{D}$ confinement systems, their morphologies cannot be observed by conventional TEM. 3D-TEM was applied to observe the frustrated morphologies of BCPs in $2 \mathrm{D}$ confinement. The $3 \mathrm{D}$ structures of poly(styrene- $b$ butadiene) (PS- $b$-PBD) in 2D confinement have been determined by 3D-TEM and are shown in Figure $4 \mathrm{a}$ as a function of $D / L_{0}{ }^{44}$ where $D$ is the diameter of cylindrical pores and $L_{0}$ is the equilibrium period of the microphase-separated structure in the bulk state. Note that this BCP forms HEX morphology in the bulk. The ratio of the two length scales, $D$ / $L_{0}$, indicates the strength of the size effect. For example, a small $D / L_{0}$ induces strong frustration for polymers. When $D / L_{0}$ is unity, torus-like nanostructures form stacked along with the long axis of the AAO cylinder (see inset). As $D / L_{0}$ increases to 1.27, a single helix of PBD is found in a PS matrix. A double helix of PBD in a PS matrix is observed at $D / L_{0}=1.6$. When $D / L_{0}$ is larger than 1.81 , cylindrical cores of PBD form in the center of the AAO cylinder, and rings $\left(D / L_{0}=1.81\right)$, a single helix $\left(D / L_{0}=2.1\right)$, and a triple helix $\left(D / L_{0}=2.2\right)$ form around the cylindrical core. Note that helical structures form in cylindrical pores that have a specific diameter even though the 
BCP has no chirality. In all morphologies, it is evident that PS, not PBD, is located at the walls of the AAO cylindrical pores, suggesting that entropic loss may occur if $\mathrm{PBD}$, the minor component, is located on the walls.

Similar frustrated morphologies from the 2D confinement have been predicted by Monte Carlo simulation. ${ }^{45}$ Figure $4 \mathrm{~b}$ shows the computer-simulated sequence of $\mathrm{BCP}$ in the $2 \mathrm{D}$ confinement as a function of $D / L_{0}$, in which the A constituent block preferentially wets the surface of the $2 \mathrm{D}$ confinement. A single-helical and torus structure appears when $D / L_{0}$ is in the range of 1.7-2.1. As $D / L_{0}$ increases to 2.2-2.4, a double helix is predicted in the $2 \mathrm{D}$ confinement. When $D / L_{0}$ is $2.9-3.3$, the morphologies of a combination of cylindrical cores and rings or single or double helix are predicted. These simulated morphologies are in good agreement with the experimental results even though $D / L_{0}$ does not exactly match. Some of the simulated morphologies are never actually observed in experiments, such as at $D / L_{0} \approx 2.7$.

A sphere $e^{46,47}$ and an inverted sphere (e.g., inverse opals ${ }^{48}$ ) have been used for $3 \mathrm{D}$ confinement. The frustrated morphologies in spherical confinement have been investigated from both experimental ${ }^{46,48,49}$ and theoretical ${ }^{50-52}$ viewpoints. Structural analysis by 3D-TEM was found to be effective for determining the actual 3D structures under this confinement because the morphologies were much more complicated than under $2 \mathrm{D}$ confinement. Figure 5 shows a comparison of the morphologies of poly(styrene- $b$-isoprene) (PS- $b$-PI) nanoparticles between the experimental results (Figure 5a) and the morphologies calculated by cell dynamics computer simulation (Figure $5 b$ ). ${ }^{53}$ The PS and PI phases are shown in blue and green, respectively. In the experimental results, a spherical particle with a biphasic structure (i.e., a Janus structure) was formed when $D / L_{0}$ was lower than 0.62 . The Janus structure is the simplest lamellar structure that forms in a nanoparticle if the particle diameter is close to the ideal thickness of a single layer of BCPs $\left(0.5 L_{0}\right)$. At $D / L_{0}=0.82$, a mushroom-like PI phase was observed, which can be considered to be a Janus-derived structure. As $D / L_{0}$ increased to 0.85 , a tennis ball-like structure formed in the particle, which is another Janus-like structure with a curved interface between the PS and PI phases. At $D / L_{0}=1.02$, a single pod was added to the tennis ball-like structure in the PI phase. As $D / L_{0}$ increased, the number of pods increased to five. This morphological series indicates that the area of the curved interfaces between PS and PI increases with $D / L_{0}$. However, the volume fraction of the PI phase $\left(f_{\mathrm{PI}}\right)$ as measured by $3 \mathrm{D}$ TEM was $0.60 \pm 0.01$, which is similar to the bulk state (i.e., 0.58 ). Thus, the density of polymers in the $3 \mathrm{D}$ confinement is expected to be similar to that in the bulk state. Some structural parameters, such as $D / L_{0}, f_{\mathrm{PI}}$, and surface areas of the nanoparticles, were quantitatively measured from the $3 \mathrm{D}$ structures obtained by 3D-TEM, which were then used for the simulation. As shown in Figure 5, good agreement between the experiments and simulations was obtained except for mismatches of the $D / L_{0}$ value. This discrepancy may be because the boundary conditions of the simulations did not perfectly reflect the actual spherical confinement. The mismatch between $D / L_{0}$ in the simulation and experiment may be caused by the fact that the boundary condition on the hard sphere in the simulation oversimplifies the experimental situation where a polymer particle is immersed in a solvent. Nonetheless, it is remarkable that the simulation produced the same sequence of structures as that of the experimental results.
As with the OOT in BCP films described in section 2, a morphological transition and transformation in 3D confinement have been reported by applying thermal annealing ${ }^{54}$ or by adding surfactant molecules to change interfacial energy between the BCP and the matrix. ${ }^{55}$ The morphological transformation between an axially stacked lamellar and onionlike structure was observed under spherical $3 \mathrm{D}$ confinement. ${ }^{54}$ Figure 6 shows the morphological transformation of lamella-
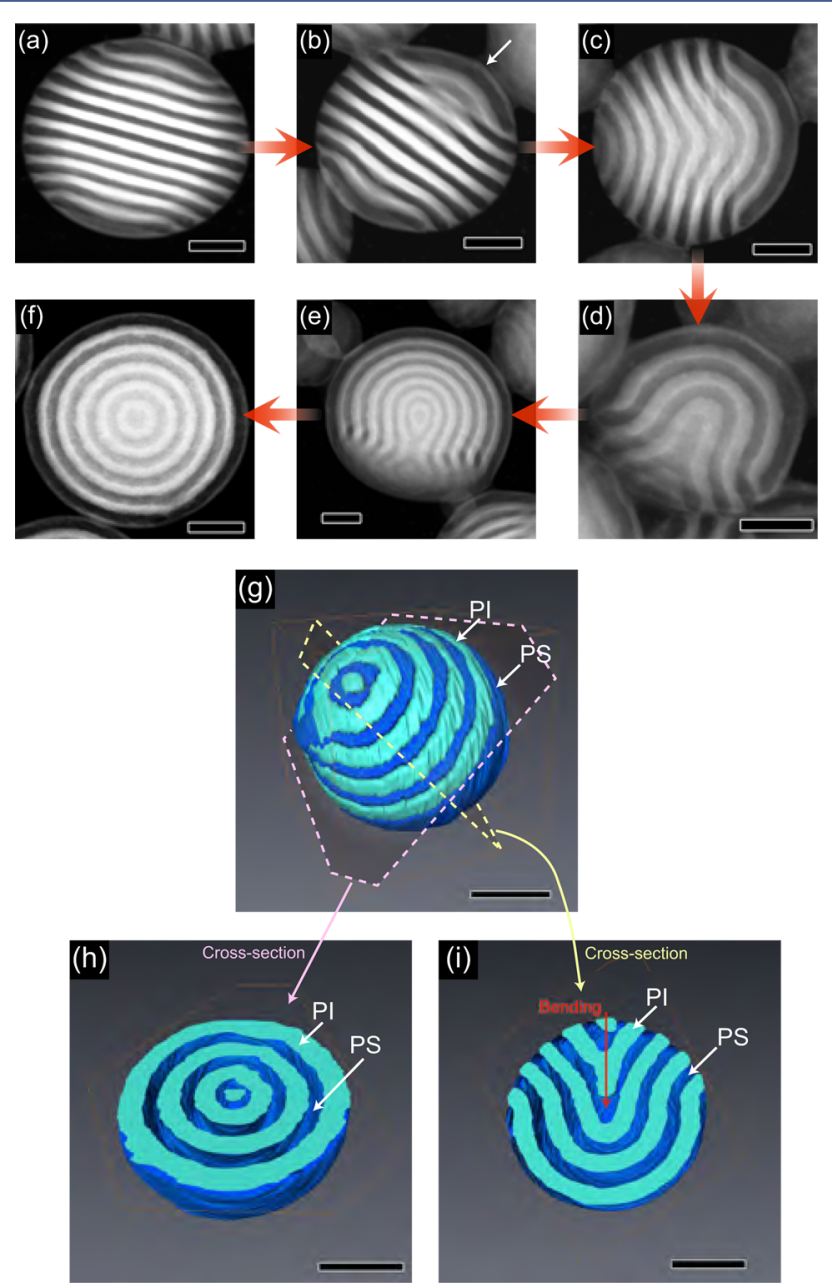

Figure 6. (a-f) ADF-STEM images of lamella-forming SI nanoparticles. The morphological transformation from stacked lamellar to onion-like structures was observed. (g) 3D structure of the nanoparticle and $(h, i)$ cross-sectional images of nanoparticles with transformed lamellar structures obtained by 3D-TEM. The blue and green regions correspond to PS and PI phases, respectively. Scale bars are $100 \mathrm{~nm}$. Adapted with permission from ref 54. Copyright 2010, Wiley-VCH Verlag GmbH \& Co.

forming SI BCP in nanoparticles dispersed in water (i.e., a poor solvent for SI) by applying thermal annealing. In the annular dark field (ADF)-STEM images, the dark and bright regions are unstained PS and PI phases stained with $\mathrm{OsO}_{4}$, respectively. As indicated by the white arrow in Figure $6 \mathrm{~b}$, the transformation starts from the polar layer of the axially stacked lamellar structure. The transformation propagates toward the opposite side (Figure 6c,d), and then the layers in the center region change to the spherical domain (Figure 6e). Eventually, all the layers transform to an onion-like structure (Figure $6 \mathrm{f}$ ). The morphology of the transformation states was observed by 3DTEM (Figure $6 \mathrm{~g}-\mathrm{i}$ ). As shown in Figure $6 \mathrm{i}$ and $\mathrm{h}$, the layer 
(a)

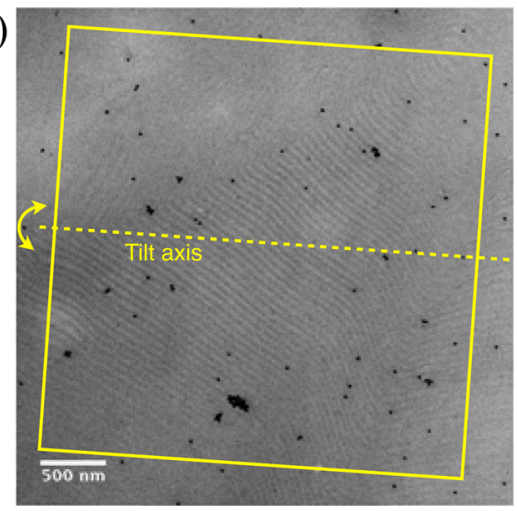

(b)

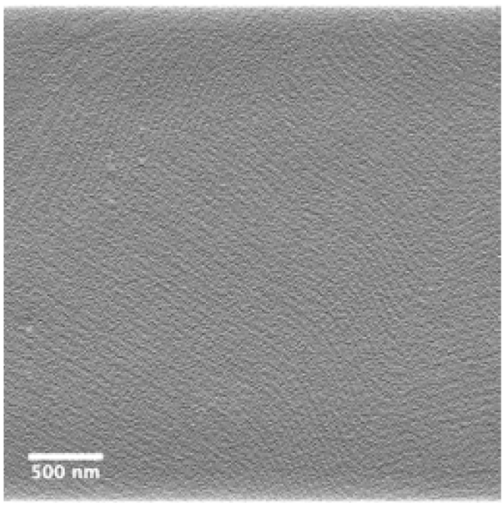

(c)

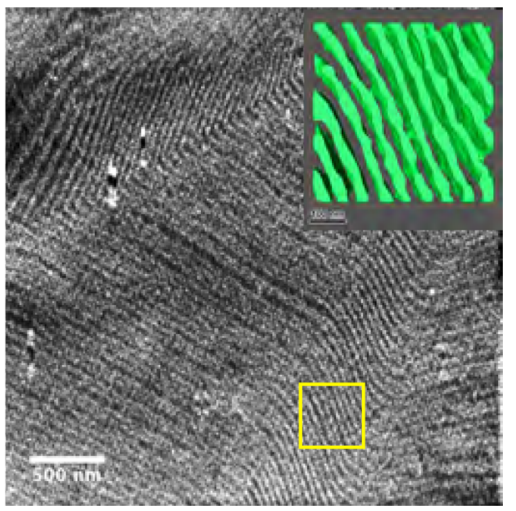

Figure 7. (a) STEM image of an unstained SI BCP using an acceleration voltage of $200 \mathrm{kV}$ with a convergence angle of $6.8 \mathrm{mrad}$. Electrons scattered up to $14.5 \mathrm{mrad}$ were used to generate the 2D STEM image. (b) Digitally sliced images reconstructed in the region enclosed by the yellow square in (a) by (b) FBP and (c) TVR-DART algorithms. For the image reconstructed by FBP, the image contrast was enhanced by the CLAHE process. ${ }^{61}$ The inset shows the $3 \mathrm{D}$ structures of PI phases visualized from the yellow square in (c).

structures become bent like a shuttlecock in the center region of the particles. For understanding the transformation process in $3 \mathrm{D}$ confinement, in situ 3D observation of a single nanoparticle provides valuable information, which is very difficult because the BCP molecules are fixed by staining.

\section{ELECTRON TOMOGRAPHY OF UNSTAINED BCP SPECIMEN TOWARD IN SITU DYNAMICAL OBSERVATION}

As mentioned in the previous sections, to understand dynamical processes, such as the self-assembly processes of BCPs, it is essential to observe structural changes in situ as a function of time. Although ultra-low-dose electron tomography with a temporal resolution of $3.5 \mathrm{~s}$ has been demonstrated in lanthanides, ${ }^{56}$ there are few studies on polymeric systems. The total accumulated dose for this in situ experiment was $8 \mathrm{e}^{-} / \AA^{2}$, which is much lower than that used for polymer 3D-TEM experiments. The requirements for in situ electron tomography in polymer research are as follows: (i) a total accumulated dose of well within several thousand electrons per square angstrom, (ii) a temporal resolution on the order of minutes or less, and (iii) an environmental setup, such as a heating stage and stretching device.

One of the most critical barriers to in situ observation is the staining protocol that is often required to enhance the contrast of the electron beam. Because most polymeric materials are composed of light elements, such as $\mathrm{C}, \mathrm{H}, \mathrm{N}$, and $\mathrm{O}$, they naturally lack electron contrast. Thus, polymers are often stained with metal regents, such as $\mathrm{OsO}_{4}$ and $\mathrm{RuO}_{4}{ }^{57}$ For example, polymers with double bonds, such as polyisoprene, can be stained with $\mathrm{OsO}_{4}$, whereas those without double bonds cannot. Therefore, in the microphase-separated structure of SI BCP, stained PI microdomains stand out from PS microdomains.

Although the staining is indeed effective for structural observations under TEM, because it fixes the morphologies, the staining protocol can be destructive for in situ dynamical imaging. We note here that the staining possibly causes slight morphological changes too. ${ }^{58}$ A method for enhancing the contrast was proposed based on the STEM. ${ }^{59}$ We also found that, with an appropriate combination of the convergence angle of electron beam and detection angle of scattered electrons of STEM, the contrast due to slight differences in density between two polymers can generate image contrast. This contrast optimization, although not regularly used, can be performed on most transmission electron microscopes. Although the previous work concluded that the contrast was generated by the inelastic scattering of electrons in their optical setup, ${ }^{59}$ in our case, the elastic scattering played a more important role. We note that other contrast enhancement approaches, such as phase plates and differential phase-contrast STEM, may be also applicable to polymers. $^{60}$

Figure $7 \mathrm{a}$ shows a STEM image from the lamellar microphase-separated structure of an unstained SI BCP in which higher density PS and lighter PI microdomains appear dark and white, respectively.

Subsequently, the STEM imaging for unstained BCP should be extended to $3 \mathrm{D}$. The area marked by a yellow square in Figure $7 \mathrm{a}$ was tilted around the axis indicated by the dotted line. STEM projections from $\pm 60^{\circ}$ with $5^{\circ}$ increment (total projections: 25) were recorded. The number of projections had to be significantly reduced compared with that of conventional electron tomography experiments, for example, 121 projections if the specimen is tilted $\pm 60^{\circ}$. This is because of the significant increase in electron beam damage to the unstained specimen, demonstrating that, in turn, the staining of one of the microphase-separated domains is an effective means of improving resistance to beam damage and thus of obtaining many projections for precise tomography experiments.

Nonetheless, 3D reconstruction using fewer STEM projections from the unstained SI BCP has been carried out by means of the conventional filtered back projection (FBP) algorithm. ${ }^{62}$ Figure $7 \mathrm{~b}$ displays one of the digitally sliced images from the $3 \mathrm{D}$ reconstruction. The lamellar microdomains can be vaguely identified. Recently, a novel algorithm for tomography was developed that can provide an accurate reconstruction from a small number of projections. Zhuge et al. demonstrated that only 21 projections over $\pm 60^{\circ}$ tilt were necessary to obtain reasonably accurate $3 \mathrm{D}$ reconstruction using their total variation regularized discrete algebraic reconstruction technique (TVR-DART), ${ }^{63,64}$ which requires prior knowledge of the number of material compositions in the specimen. This new reconstruction algorithm has been applied to the same tilt series of the same data set for the unstained BCP sample (in Figure $7 a$ ). Figure $7 \mathrm{c}$ displays the digitally sliced image from the $3 \mathrm{D}$ reconstruction using TVR-DART at exactly the same position of the reconstruction shown in Figure $7 \mathrm{~b}$. Distinctive differences between the two reconstruction algorithms can be 
seen with the lamellar microdomains now clearly identified in Figure 7c. Segmentation of one of the microdomains (PI domains) was possible, and the resulting surface-rendered 3D image can be generated (see inset of Figure 7c). We note that the total electron dose used to obtain the $3 \mathrm{D}$ reconstruction in Figure $7 \mathrm{~b}$ and $\mathrm{c}$ was roughly $1 / 70$ th of that used under normal experimental conditions for $3 \mathrm{D} \mathrm{BCP}$ imaging.

\section{CONCLUSIONS}

Electron tomography, a nanoscale 3D visualization method, has been used to investigate self-assembling nanostructures and their self-assembly processes in polymer systems. BCPs were chosen as a representative example of this. The OOT between DG and HEX nanostructures was studied in which certain epitaxy has been found. The epitaxial relationship differed depending on the direction of the OOT, which is still an unsolved issue. Another type of OOT between cylinder and HPL structures was also studied in which the HPL layers were found to alternately connect to one of two nonintersecting DG networks. These epitaxial relationships in OOTs could be induced not only by temperature but also by applying an external field, such as a shear flow or electric field. Therefore, these phenomena are important for the industrial design of products as well as for academic understanding of dynamical processes as typical examples of nonequilibrium and nonlinear mechanics. We note that the above experiments have been carried out using specimens frozen during OOTs, and the boundaries between the two coexisting phases were analyzed by electron tomography. Although electron tomography is found to be the most effective visualization tool for observing the dynamical processes during OOT, in situ direct $3 \mathrm{D}$ imaging is preferable. $^{56}$

BCP morphologies under confinement are also interesting because even BCPs with simple morphologies in the bulk, such as lamellar structures, can exhibit very complicated morphologies. $D / L_{0}$ as well as the surface energy of $B C P$ s to the confinement wall are key parameters for controlling these complicated morphologies. The obtained 3D images were compared with results from computer simulations. Although not completely quantitative, excellent agreement was obtained between the experiments and simulations. Dynamical processes of BCP under confinement are interesting but remain open issues.

Although electron tomography has been shown to be quite useful for direct imaging and obtaining both static and dynamical structural information at the nanoscale, few studies have reported in situ dynamical processes, such as morphological changes in OOTs as a function of time in the same area of a specimen. In polymeric samples, it is particularly difficult because of the staining protocol and electron beam damage to the polymers. As a prelude to in situ electron tomography, a method for enhancing contrast from the unstained BCP sample based on STEM (Figure 7a) was developed. Contrast occurs because of subtle differences in density between the constituent polymers due to chemical structure differences. A novel 3D reconstruction algorithm was developed and used together with the contrast enhancement method for STEM, which allowed us to perform 3D imaging of beam-sensitive unstained polymer specimens (Figure 7c). This novel low-dose electron tomography may provide a method for in situ electron tomography in the near future.

\section{ASSOCIATED CONTENT}

\section{Supporting Information}

The Supporting Information is available free of charge on the ACS Publications website at DOI: 10.1021/acs.accounts.7b00103.

Movie of the 3D structure of poly(styrene- $b$-isoprene) during order-order transition from hexagonally perforated lamellar to double gyroid structures, which corresponds to Figure 3b (MPG)

\section{AUTHOR INFORMATION}

\section{Corresponding Author}

*E-mail: hjinnai@tagen.tohoku.ac.jp; phone: +81-22-217-5329; fax: +81-22-217-5329.

ORCID $\odot$

Hiroshi Jinnai: 0000-0003-3400-1928

Notes

The authors declare no competing financial interest.

\section{Biographies}

Hiroshi Jinnai is a Professor of the Institute of Multidisciplinary Research for Advanced Materials (IMRAM), Tohoku University. He received his D.Eng. from Kyoto University, Japan, in 1993. His research fields include polymer physics, especially in the area of phase transitions of polymer alloys, and THE development of electron microscopy methodologies. He is the recipient of several awards, including the Ernst-Ruska-Prize from Deutsche Gesellschaft für Elektronenmikroskopie e.V. (2007), the Japanese Society of Microscopy Society Award, the Setoh Prize (2012), and the award of the The Society of Polymer Science, Japan (2017). He has been a Fellow of the American Physical Society since 2011.

Takeshi Higuchi received his Ph.D. from Hokkaido University in 2008. He has been an Assistant Professor of IMRAM, Tohoku University, since 2013. His research focuses on structural analysis of polymer assemblies based on electron microscopy.

Xiaodong Zhuge is a Senior Researcher at Centrum Wiskunde \& Informatica (CWI), the national research institute for mathematics and computer science in The Netherlands. His research interests include methods, algorithms, and numerical solutions for large-scale inverse problems in imaging and tomography under limited data conditions.

Akihito Kumamoto is currently a Senior Researcher at the University of Tokyo, Japan. His research interests include industrial applications of aberration-corrected (scanning) transmission electron microscopy and atomic resolution chemical mapping using energy-dispersive $\mathrm{X}$-ray spectroscopy.

kees Joost Batenburg leads the Computational Imaging group at CWI, Amsterdam. He is also a part-time full professor at Leiden University. His research interests include a broad range of 3D image reconstruction problems, in particular, problems related to tomography.

Yuichi Ikuhara is the Director of the Nanotechnology Center, Institute of Engineering Innovation, at the University of Tokyo. He received his D.Eng. from the Materials Sciences Department of Kyushu University and joined the Japan Fine Ceramics Center in 1988. He has received the "Medal with Purple Ribbon", the "Humboldt Research Award", and other awards. He is a fellow of the American Ceramics Society. 


\section{ACKNOWLEDGMENTS}

We thank Prof. T. Chang and his group members (Pohang University of Science and Technology, Korea) for their support, collaboration, and useful discussions. This work was partially supported by JSPS KAKENHI Grant Numbers $16 \mathrm{H} 06040,16 \mathrm{H} 02288$, and 16K14001 Japan. This work was also performed under the Cooperative Research Program of "Network Joint Research Center for Materials and Devices."

\section{REFERENCES}

(1) Yang, S. Y.; Ryu, I.; Kim, H. Y.; Kim, J. K.; Jang, S. K.; Russell, T. $\mathrm{P}$. Nanoporous membranes with ultrahigh selectivity and flux for the filtration of viruses. Adv. Mater. 2006, 18, 709-712.

(2) Leong, W. L.; Lee, P. S.; Lohani, A.; Lam, Y. M.; Chen, T.; Zhang, S.; Dodabalapur, A.; Mhaisalkar, S. G. Non-Volatile Organic Memory Applications Enabled by In Situ Synthesis of Gold Nanoparticles in a Self-Assembled Block Copolymer. Adv. Mater. 2008, 20, 2325-2331.

(3) Zhao, Y.; Thorkelsson, K.; Mastroianni, A. J.; Schilling, T.; Luther, J. M.; Rancatore, B. J.; Matsunaga, K.; Jinnai, H.; Wu, Y.; Poulsen, D.; Frechet, J. M. J.; Alivisatos, A. P.; Xu, T. Small-moleculedirected nanoparticle assembly towards stimuli-responsive nanocomposites. Nat. Mater. 2009, 8, 979-985.

(4) Li, Z.; Hur, K.; Sai, H.; Higuchi, T.; Takahara, A.; Jinnai, H.; Gruner, S. M.; Wiesner, U. Linking experiment and theory for threedimensional networked binary metal nanoparticle-triblock terpolymer superstructures. Nat. Commun. 2014, 5, 3247.

(5) Buban, J. P.; Matsunaga, K.; Chen, J.; Shibata, N.; Ching, W. Y.; Yamamoto, T.; Ikuhara, Y. Grain Boundary Strengthening in Alumina by Rare Earth Impurities. Science 2006, 311, 212-215.

(6) Jinnai, H.; Nishikawa, Y.; Koga, T.; Hashimoto, T. Direct Observation of 3-Dimensional Bicontinuous Structure Developed via Spinodal Decomposition. Macromolecules 1995, 28, 4782-4784.

(7) Jinnai, H.; Koga, T.; Nishikawa, Y.; Hashimoto, T.; Hyde, S. T. Curvature determination of spinodal interface in a condensed matter system. Phys. Rev. Lett. 1997, 78, 2248-2251.

(8) Jinnai, H.; Nishikawa, Y.; Morimoto, H.; Koga, T.; Hashimoto, T. Geometrical Properties and Interface Dynamics: Time Evolution of Spinodal Interface in a Binary Polymer Mixture at the Critical Composition. Langmuir 2000, 16, 4380-4393.

(9) Saito, H.; Aoyanagi, Y.; Mihara, T.; Tanaka, T.; Higuchi, T.; Morita, H.; Jinnai, H. Direct three-dimensional imaging of the fracture of fiber-reinforced plastic under uniaxial extension: Effect of adhesion between fibers and matrix. Polymer 2017, 116, 556.

(10) Miller, M. K. Atom-probe Tomography: Analysis at the Atomic Level; Kruwer Academic/Plenum Press: New York, 2000.

(11) Kawase, N.; Kato, M.; Nishioka, H.; Jinnai, H. Transmission Electron Microtomography without the "Missing Wedge" for Quantitative Structural Analysis. Ultramicroscopy 2007, 107, 8-15.

(12) Jinnai, H.; Spontak, R. J. Transmission Electron Microtomography in Polymer Research. Polymer 2009, 50, 1067-1087.

(13) Jinnai, H.; Spontak, R. J.; Nishi, T. Transmission Electron Microtomography and Polymer Nanostructures. Macromolecules 2010, 43, 1675-1688.

(14) Jinnai, H.; Watashiba, H.; Kajihara, T.; Takahashi, M. Connectivity and topology of a phase-separating bicontinuous structure in a polymer mixture: Direct measurements of coordination number, inter-junction distances and Euler characteristic. J. Chem. Phys. 2003, 119, 7554-7559.

(15) Spontak, R. J.; Williams, M. C.; Agard, D. A. Three-dimensional study of cylindrical morphology in a styrene-butadiene-styrene block copolymer. Polymer 1988, 29, 387-395.

(16) Schulz, M. F.; Bates, F. S.; Almdal, K.; Mortensen, K. Epitaxial Relationship for Hexagonal-to-Cubic Phase Transition in a Book Copolymer Mixture. Phys. Rev. Lett. 1994, 73, 86-89.

(17) Zhao, J.; Majumdar, B.; Schulz, M. F.; Bates, F. S.; Almdal, K.; Mortensen, K.; Hajduk, D. A.; Gruner, S. M. Phase Behavior of Pure
Diblocks and Binary Diblock Blends of Poly(ethylene)-Poly(ethylethylene). Macromolecules 1996, 29, 1204-1215.

(18) Vigild, M. E.; Almdal, K.; Mortensen, K.; Hamley, I. W.; Fairclough, J. P. A.; Ryan, A. J. Transformations to and from the Gyroid Phase in a Diblock Copolymer. Macromolecules 1998, 31, 5702-5716.

(19) Matsen, M. W. Cylinder $\leftrightarrow$ Gyroid Epitaxial Transitions in Complex Polymeric Liquids. Phys. Rev. Lett. 1998, 80, 4470-4473.

(20) Kimishima, K.; Koga, T.; Hashimoto, T. Order-Order Phase Transition between Spherical and Cylindrical Microdomain Structures of Block Copolymer. I. Mechanism of the Transition. Macromolecules 2000, 33, 968-977.

(21) Wang, C.-Y.; Lodge, T. P. Kinetics and Mechanisms for the Cylinder-to-Gyroid Transition in a Block Copolymer Solution. Macromolecules 2002, 35, 6997-7006.

(22) Park, I.; Lee, B.; Ryu, J.; Im, K.; Yoon, J.; Ree, M.; Chang, T. Epitaxial Phase Transition of Polystyrene-b-Polyisoprene from Hexagonally Perforated Layer to Gyroid Phase in Thin Film. Macromolecules 2005, 38, 10532-10536.

(23) Honda, T.; Kawakatsu, T. Epitaxial Transition from Gyroid to Cylinder in a Diblock Copolymer Melt. Macromolecules 2006, 39, 2340-2349.

(24) Wang, C.; Jiang, K.; Zhang, P.; Shi, A.-C. Origin of epitaxies between ordered phases of block copolymers. Soft Matter 2011, 7, 10552-10555.

(25) Cheng, X.; Lin, L.; Weinan, E.; Zhang, P.; Shi, A. C. Nucleation of ordered phases in block copolymers. Phys. Rev. Lett. 2010, 104, 148301.

(26) Park, H.-W.; Jung, J.; Chang, T.; Matsunaga, K.; Jinnai, H. New Epitaxial Phase Transition between DG and HEX in PS-b-PI. J. Am. Chem. Soc. 2009, 131, 46-47.

(27) Sugimori, H.; Niihara, K.; Kaneko, T.; Miyoshi, W.; Jinnai, H. Direct Three-Dimensional Observations of Order-Order Transition from Gyroid to Cylindrical Structures in a Block Copolymer. Progr. Theor. Phys. Suppl. 2008, 175, 166-173.

(28) Jung, J.; Lee, J.; Park, H.-W.; Chang, T.; Sugimori, H.; Jinnai, H. Epitaxial Phase Transition between Double Gyroid and Cylinder Phase in Diblock Copolymer Thin Film. Macromolecules 2014, 47, $8761-8767$.

(29) Hamley, I. W.; Koppi, K. A.; Rosedale, J. H.; Bates, F. S.; Almdal, K.; Mortensen, K. Hexagonal Mesophases Between Lamellae and Cylinders in a Diblock Copolymer Melt. Macromolecules 1993, 26, 5959-5970.

(30) Dohi, H.; Kimura, H.; Kotani, M.; Kaneko, T.; Kitaoka, T.; Nishi, T.; Jinnai, H. Three-Dimensional Imaging in Polymer Science: Its Application to Block Copolymer Morphologies and Rubber Composites. Polym. J. 2007, 39, 749-758.

(31) Mareau, V. H.; Akasaka, S.; Osaka, T.; Hasegawa, H. Direct Visualization of the Perforated Layer/Gyroid Grain Boundary in a Polystyrene-block-polyisoprene Blend by Electron Tomography. Macromolecules 2007, 40, 9032-9039.

(32) Shi, A.-C.; Li, B. Self-assembly of diblock copolymers under confinement. Soft Matter 2013, 9, 1398-1413.

(33) Yabu, H.; Higuchi, T.; Jinnai, H. Frustrated phases: polymeric self-assemblies in a 3D confinement. Soft Matter 2014, 10, 2919-2931.

(34) Jeong, S.-J.; Xia, G.; Kim, B. H.; Shin, D. O.; Kwon, S.-H.; Kang, S.-W.; Kim, S. O. Universal Block Copolymer Lithography for Metals, Semiconductors, Ceramics, and Polymers. Adv. Mater. 2008, 20, $1898-1904$

(35) Cheng, J. Y.; Ross, C. A.; Smith, H. I.; Thomas, E. L. Templated Self-Assembly of Block Copolymers: Top-Down Helps Bottom-Up. Adv. Mater. 2006, 18, 2505-2521.

(36) Bang, J.; Bae, J.; Lowenhielm, P.; Spiessberger, C.; Given-Beck, S. A.; Russell, T. P.; Hawker, C. J. Facile routes to patterned surface neutralization layers for block copolymer lithography. Adv. Mater. 2007, 19, 4552-4457.

(37) Russell, T. P.; Menelle, A.; Anastasiadis, S.; Satija, S.; Majkrzak, C. Unconventional morphologies of symmetric, diblock copolymers 
due to film thickness constraints. Macromolecules 1991, 24, 62636269.

(38) Knoll, A.; Horvat, A.; Lyakhova, K.; Krausch, G.; Sevink, G.; Zvelindovsky, A. V.; Magerle, R. Phase Behavior in Thin Films of Cylinder-Forming Block Copolymers. Phys. Rev. Lett. 2002, 89, 035501.

(39) Niihara, K.-I.; Sugimori, H.; Matsuwaki, U.; Hirato, F.; Morita, H.; Doi, M.; Masunaga, H.; Sasaki, S.; Jinnai, H. A Transition from Cylindrical to Spherical Morphology in Diblock Copolymer Thin Films. Macromolecules 2008, 41, 9318-9325.

(40) Ma, M.; Krikorian, V.; Yu, J. H.; Thomas, E. L.; Rutledge, G. C. Electrospun Polymer Nanofibers with Internal Periodic Structure Obtained by Microphase Separation of Cylindrically Confined Block Copolymers. Nano Lett. 2006, 6, 2969-2972.

(41) Kalra, V.; Mendez, S.; Lee, J. H.; Nguyen, H.; Marquez, M.; Joo, Y. L. Confined Assembly in Coaxially Electrospun Block Copolymer Fibers. Adv. Mater. 2006, 18, 3299-3303.

(42) Shin, K.; Xiang, H.; Moon, S.; Kim, T.; McCarthy, T.; Russell, T. P. Curving and frustrating flatland. Science 2004, 306, 76-76.

(43) Xiang, H.; Shin, K.; Kim, T.; Moon, S.; McCarthy, T. J.; Russell, $\mathrm{T}$. P. The influence of confinement and curvature on the morphology of block copolymers. J. Polym. Sci., Part B: Polym. Phys. 2005, 43, 3377-3383.

(44) Dobriyal, P.; Xiang, H.; Kazuyuki, M.; Chen, J.-T.; Jinnai, H.; Russell, T. P. Cylindrically Confined Diblock Copolymers. Macromolecules 2009, 42, 9082-9088.

(45) Yu, B.; Jin, Q.; Ding, D.; Li, B.; Shi, A.-C. Confinement-Induced Morphologies of Cylinder-Forming Asymmetric Diblock Copolymers. Macromolecules 2008, 41, 4042-4054.

(46) Higuchi, T.; Motoyoshi, K.; Sugimori, H.; Jinnai, H.; Yabu, H.; Shimomura, M. Three-dimensional observation of confined phaseseparated structures in block copolymer nanoparticles. Soft Matter 2012, 8, 3791-3797.

(47) Wyman, I.; Njikang, G.; Liu, G. When emulsification meets selfassembly: The role of emulsification in directing block copolymer assembly. Prog. Polym. Sci. 2011, 36, 1152-1183.

(48) Rider, D. A.; Chen, J. I. L.; Eloi, J.-C.; Arsenault, A. C.; Russell, T. P.; Ozin, G. A.; Manners, I. Controlling the Morphologies of Organometallic Block Copolymers in the 3-Dimensional Spatial Confinement of Colloidal and Inverse Colloidal Crystals. Macromolecules 2008, 41, 2250-2259.

(49) Arsenault, A. C.; Rider, D. A.; Tetreault, N.; Chen, J. I. L.; Coombs, N.; Ozin, G. A.; Manners, I. Block copolymers under periodic, strong three-dimensional confinement. J. Am. Chem. Soc. 2005, 127, 9954-9955.

(50) Sevink, G. J. A.; Zvelindovsky, A. V. Self-Assembly of Complex Vesicles. Macromolecules 2005, 38, 7502-7513.

(51) Yu, B.; Li, B.; Jin, Q.; Ding, D.; Shi, A.-C. Self-Assembly of Symmetric Diblock Copolymers Confined in Spherical Nanopores. Macromolecules 2007, 40, 9133-9142.

(52) Yang, R.; Li, B.; Shi, A.-C. Phase Behavior of Binary Blends of Diblock Copolymer/Homopolymer Confined in Spherical Nanopores. Langmuir 2012, 28, 1569-1578.

(53) Higuchi, T.; Pinna, M.; Zvelindovsky, A. V.; Jinnai, H.; Yabu, H. Multipod structures of lamellae-forming diblock copolymers in threedimensional confinement spaces: Experimental observation and computer simulation. J. Polym. Sci., Part B: Polym. Phys. 2016, 54, $1702-1709$.

(54) Higuchi, T.; Motoyoshi, K.; Sugimori, H.; Jinnai, H.; Yabu, H.; Shimomura, M. Phase Transition and Phase Transformation in Block Copolymer Nanoparticles. Macromol. Rapid Commun. 2010, 31, $1773-1778$.

(55) Klinger, D.; Wang, C. X.; Connal, L. A.; Audus, D. J.; Jang, S. G.; Kraemer, S.; Killops, K. L.; Fredrickson, G. H.; Kramer, E. J.; Hawker, C. J. A Facile Synthesis of Dynamic, Shape-Changing Polymer Particles. Angew. Chem., Int. Ed. 2014, 53, 7018-7022.

(56) Migunov, V.; Ryll, H.; Zhuge, X.; Simson, M.; Strüder, L.; Batenburg, K. J.; Houben, L.; Dunin-Borkowski, R. E. Rapid low dose electron tomography using a direct electron detection camera. Sci. Rep. 2015, 5, 14516.

(57) Kato, K. Electron Microscopy of ABS Plastics. J. Electron Microsc. $1965,14,220-221$.

(58) Ishige, R.; Higuchi, T.; Jiang, X.; Mita, K.; Ogawa, H.; Yokoyama, H.; Takahara, A.; Jinnai, H. Structural Analysis of Microphase Separated Interface in an ABC-Type Triblock Terpolymer by Combining Methods of Synchrotron-Radiation Grazing Incidence Small-Angle X-ray Scattering and Electron Microtomography. Macromolecules 2015, 48, 2697-2705.

(59) Aso, R.; Kurata, H.; Namikoshi, T.; Hashimoto, T.; Kuo, S.-W.; Chang, F.-C.; Hasegawa, H.; Tsujimoto, M.; Takano, M.; Isoda, S. Quantitative Imaging of T-g in Block Copolymers by Low-Angle Annular Dark-Field Scanning Transmission Electron Microscopy. Macromolecules 2013, 46, 8589-8595.

(60) Danev, R.; Nagayama, K. Transmission electron microscopy with Zernike phase plate. Ultramicroscopy 2001, 88, 243-252.

(61) Zuiderveld, K. In Graphics Gems IV; Heckbert, P. S., Ed.; Academic Press Professional, Inc.: San Diego, CA, USA, 1994; Chapter Contrast Limited Adaptive Histogram Equalization, pp 474485 .

(62) Crowther, R. A.; DeRosier, D. J.; Klug, A. The Reconstruction of a Three-Dimensional Structure from Projections and its Application to Electron Microscopy. Proc. R. Soc. London, Ser. A 1970, 317, 319340.

(63) Zhuge, X.; Palenstijn, W.; Batenburg, K. TVR-DART: A More Robust Algorithm for Discrete Tomography From Limited Projection Data With Automated Gray Value Estimation. IEEE Trans. Image Process. 2016, 25, 455-468.

(64) Zhuge, X.; Jinnai, H.; Dunin-Borkowski, R. E.; Migunov, V.; Bals, S.; Cool, P.; Bons, A.-J.; Batenburg, K. J. Automated discrete electron tomography - Towards routine high-fidelity reconstruction of nanomaterials. Ultramicroscopy 2017, 175, 87-96. 